\title{
A large focus of naturally acquired Plasmodium knowlesi infections in human beings
}

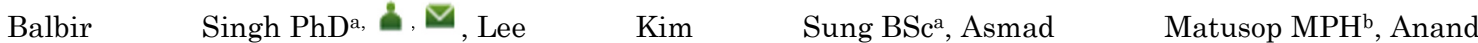 \\ Radhakrishnan MSc ${ }^{a}$, Sunita SG Shamsul BSc ${ }^{a}$, Janet Cox-Singh $\mathrm{PhD}^{\mathrm{a}}$, Alan ThomasPhD ${ }^{\mathrm{c}}$, David J \\ Conway $\mathrm{PhD}^{\mathrm{d}}$ \\ a Faculty of Medicine and Health Sciences, Universiti Malaysia Sarawak, Kuching, Sarawak, Malaysia \\ b Sarawak State Health Department, Kuching, Sarawak \\ c Department of Parasitology, Biomedical Primate Research Centre, Rijswijk, Netherlands \\ d Department of Infectious and Tropical Diseases, London School of Hygiene and Tropical Medicine, \\ London, UK
}

\section{Summary}

\section{Background}

About a fifth of malaria cases in 1999 for the Kapit division of Malaysian Borneo had routinely been identified by microscopy as Plasmodium malariae, although these infections appeared atypical and a nested PCR assay failed to identify $P$ malariae DNA. We aimed to investigate whether such infections could be attributable to a variant form of $P$ malariae or a newly emergent Plasmodium species.

\section{Methods}

We took blood samples from 208 people with malaria in the Kapit division between March, 2000, and November, 2002. The small subunit ribosomal RNA and the circumsporozoite protein genes were sequenced for eight isolates that had been microscopically identified as $P$ malariae. All blood samples were characterised with a genus-specific and species-specific nested PCR assay together with newly designed $P$ knowlesi-specific primers.

\section{Findings}

All DNA sequences were phylogenetically indistinguishable from those of $P$ knowlesi, a malaria parasite of long-tailed macaque monkeys, but were significantly different from other malaria parasite species. By PCR assay, 120 (58\%) of 208 people with malaria tested positive for $P$ knowlesi, whereas none was positive for $P$ malariae. $P$ knowlesi parasites in human erythrocytes were difficult to distinguish from $P$ malariae by microscopy. Most of the $P$ knowlesi infections were in adults and we did not note any clustering of cases within communities. $P$ knowlesi infections were successfully treated with chloroquine and primaquine.

\section{Interpretation}

Naturally acquired $P$ knowlesi infections, misdiagnosed by microscopy mainly as $P$ malariae, accounted for over half of all malaria cases in our study. Morphological similarities between $P$ knowlesi and $P$ malariae necessitate the use of molecular methods for correct identification. Further work is needed to determine whether human $P$ knowlesi infections in the Kapit division are acquired from macaque monkeys or whether a host switch to human beings has occurred.

Published in The Lancet, Volume 363, Issue 9414, 27 March 2004, Pages 1017-1024 